Revue d'histoire de l'Amérique française

Q6 REVUE D.HISTOIRE DE L'AMÉRIQUE FRANÇAISE

\title{
Les stratégies agricoles du paysan canadien-français de l'est ontarien (1870)
}

\section{Nicole Castéran}

Volume 41, numéro 1, été 1987

URI : https://id.erudit.org/iderudit/304521ar

DOI : https://doi.org/10.7202/304521ar

Aller au sommaire du numéro

\section{Éditeur(s)}

Institut d'histoire de l'Amérique française

\section{ISSN}

0035-2357 (imprimé)

1492-1383 (numérique)

Découvrir la revue

\section{Citer cet article}

Castéran, N. (1987). Les stratégies agricoles du paysan canadien-français de l'est ontarien (1870). Revue d'histoire de l'Amérique française, 41(1), 23-51. https://doi.org/10.7202/304521ar

\section{Résumé de l'article}

Fondée essentiellement sur les données du recensement de 1871, cette étude compare les stratégies agricoles mises en oeuvre par des agriculteurs canadiens-français et canadiens-anglais du comté de Prescott, Ontario. L'approche écologique utilisée a pour but de mettre en relief les influences de l'environnement physique et du milieu social sur le choix de la stratégie.

Nous avons sélectionné un ensemble de 68 fermes en fonction de l'origine ethnique du chef de famille et du type de sol sur lequel elles étaient situées. Des méthodes d'analyse multivariées - une analyse en composantes principales et une analyse de groupement - ont permis d'établir une typologie des fermes en fonction de critères purement agronomiques. Nous avons ainsi obtenu quatre principaux groupes se distinguant essentiellement par la productivité et le type d'utilisation du sol.

Ces groupes de fermes ont été mis en relation avec les facteurs édaphique et ethnique jusque-là volontairement tenus à l'écart. La répartition des types de sol et des ethnies à l'intérieur de chacun des groupes a clairement démontré l'influence déterminante qu'exercent ces facteurs sur le choix des stratégies agricoles. Les Canadiens français se sont avérés plus nombreux dans les deux groupes affichant la plus faible productivité. Cette relation a pris tout son sens à la lumière de diverses propriétés du sol telles la fertilité et la maniabilité et de certains traits démographiques et économiques prévalant chez les Canadiens français du comté de Prescott. En effet, la force de travail limitée et le niveau de richesse généralement bas qui les caractérisent, conjugués à certaines caractéristiques du sol, n'autorisent pas la mise en oeuvre d'une stratégie visant à une maximisation de la production.
Tous droits réservés @ Institut d'histoire de l'Amérique française, 1987
Ce document est protégé par la loi sur le droit d'auteur. L'utilisation des services d'Érudit (y compris la reproduction) est assujettie à sa politique d'utilisation que vous pouvez consulter en ligne.

https://apropos.erudit.org/fr/usagers/politique-dutilisation/ 


\title{
LES STRATÉGIES AGRICOLES DU PAYSAN CANADIEN-FRANÇAIS DE L'EST ONTARIEN $(1870)^{1}$
}

\author{
NICOLE CASTÉRAN \\ Ottawa
}

\begin{abstract}
RÉSUMÉ
Fondée essentiellement sur les données du recensement de 1871, cette étude compare les stratégies agricoles mises en oeuvre par des agriculteurs canadiens-français et canadiens-anglais du comté de Prescott, Ontario. L'approche écologique utilisée a pour but de mettre en relief les influences de l'environnement physique et du milieu social sur le choix de la stratégie.
\end{abstract}

Nous avons sélectionné un ensemble de 68 fermes en fonction de l'origine ethnique du chef de famille et du type de sol sur lequel elles étaient situées. Des méthodes d'analyse multivariées - une analyse en composantes principales et une analyse de groupement — ont permis d'établir une typologie des fermes en fonction de critères purement agronomiques. Nous avons ainsi obtenu quatre principaux groupes se distinguant essentiellement par la productivité et le type d'utilisation du sol.

Ces groupes de fermes ont été mis en relation avec les facteurs édaphique et ethnique jusquelà volontairement tenus à l'écart. La répartition des types de sol et des ethnies à l'intérieur de chacun des groupes a clairement démontré l'influence déterminante qu'exercent ces facteurs sur le choix des stratégies agricoles. Les Canadiens français se sont avérés plus nombreux dans les deux groupes affichant la plus faible productivité. Cette relation a pris tout son sens à la lumière de diverses propriétés du sol telles la fertilité et la maniabilité et de certains traits démographiques et économiques prévalant chez les Canadiens français du comté de Prescott. En effet, la force de travail limitée et le niveau de richesse généralement bas qui les caractérisent, conjugués à certaines caractéristiques du sol, n'autorisent pas la mise en oeuvre d'une stratégie visant à une maximisation de la production.

\begin{abstract}
Based mainly on the 1871 census, this study compares the farming practices of anglophones and francophones in Prescott county, Ontario. The ecological approach followed here identifies both environmental and social factors which influenced the choice of agricultural strategies by these two ethnic groups.
\end{abstract}

A sample of 68 farms has been selected according to ethnic origin and type of soil. Multivariate analysis methods (a principal component analysis and a cluster analysis) have led to the

1 Cet article est tiré de notre thèse de maîtrise «Écologie et agriculture pré-industrielle dans l'est ontarien» présentée en 1985 au département de Géographie de l'Université d'Ottawa. Nous tenons à exprimer notre gratitude à notre directeur de thèse Michel Phipps ainsi qu'aux organismes qui ont subventionné la recherche, soit l'École des études supérieures de l'Université d'Ottawa, le Régime de bourses de l'enseignement supérieur de l'Ontario et le Centre de recherches en civilisation canadienne-française. Nous remercions enfin Jean-Pierre Hardy et René Hardy pour leurs précieux commentaires.

RHAF, vol. 41, no 1, été 1987 
creation of a typology of these farms according to purely agronomic criteria. Four main categories of farms have emerged, characterized by their productivity and land use.

These groups have then been analyzed in light of edaphic and ethnic factors, which have been kept intentionally in the background. The distribution of soil types and ethnic characteristics by farm category has demonstrated clearly the decisive influence of these factors over the choice of agricultural strategies. French Canadians appeared to be more numerous in the two groups with the lowest productivity. This relationship can be explained in light of various environmental properties such as fertility and workability of the soil and of the demographic and economic features of the French Canadians of Prescott county. Indeed, limited manpower and a generally low income, in addition to special soil characteristics, have hindered the implementation of a productivity-oriented strategy by francophones.

\section{INTRODUCTION}

Le paysan canadien-français du $19 \mathrm{e}$ siècle a longtemps été dépeint sous les traits d'un personnage enfermé dans les traditions et aveugle au progrès. Son type d'agriculture peu progressiste contrastait, semble$t$-il, avec celui de ses voisins anglophones ${ }^{2}$. A la suite des travaux de Wallot et Paquet ${ }^{3}$, des études de plus en plus nombreuses tentent de démontrer que cette conception traditionnelle de l'habitant canadienfrançais traduit mal la réalité révélée par certains documents; en effet, le portrait d'un agriculteur plus entreprenant se profile peu à peu ${ }^{4}$.

₹ Pour Ouellet, et c'est là l'essence même de sa thèse, le conservatisme incurable des Canadiens français est le grand responsable du sous-développement économique de ce peuple, $\mathrm{F}$. Ouellet, «Libéré ou exploité! Le paysan québécois d'avant 1850», Histoire sociale, 13,26 (1980): 339-368; «Le mythe de l'habitant sensible au marché», Recherches sociographiques, 17 (1976): 115-132. En fait, Ouellet reprend, en l'étayant sur des données statistiques, une des idées de Jones qui considérait que «It was this aversion to innovation which rendered the distress in the seigneuries so acute». R. L. Jones, «French Canadian agriculture in the Saint-Lawrence Valley, 18151850», Agricultural History, 16 (1942): 137-141.

Farouchement opposés à ces idées, Wallot et Paquet considèrent plutôt le cultivateur canadien-français comme un agent économique rationnel qui ne modifie pas ses méthodes de culture parce qu'il est plus rentable de défricher de nouvelles terres et qu'en outre il ne dispose pas des capitaux nécessaires à une modernisation. Voir particulièrement «Crise agricole et tensions socio-ethniques dans le Bas-Canada, 1802-1812: éléments pour une réinterprétation», Revue d'histoire de l'Amérique française, 26,2 (septembre 1972): 185-237; «Stratégie foncière de l'habitant: Québec (1790-1835)», RHAF, 39,4 (printemps 1986): 551-581.

4 Que l'on songe par exemple aux travaux de F. Lewis et M. McInnis qui ont comparé l'efficacité agricole dans 90 comtés du Bas-Canada en 1851 sans qu'il soit possible de déceler un quelconque retard de la part des Canadiens français, «The Efficiency of the French Canadian Farmer in the XIXe century», Journal of Economic History, 40 (1980): 497-514; «Agricultural Output and Efficiency in Lower Canada, 1851», Research in Economic History, 9 (1984): 45-87. Mentionnons l'étude de J. McCallum qui attribue le retard économique du Québec par rapport à celui de l'Ontario dans la seconde moitié du $19 \mathrm{e}$ siècle à des conditions physiques moins favorables à l'agriculture et à une conjoncture économique continentale tendant à favoriser les régions de l'ouest au détriment du Québec et de la Nouvelle-Angleterre, Unequal Beginning (Toronto, University of Toronto Press, 1980). Mentionnons aussi celles de S. Courville qui montre que la parcellisation du terroir, la diversité des rendements et l'abandon de la culture du blé correspondent à un dynamisme d'adaptation vis-à-vis d'une nouvelle conjoncture, «La crise agricole du BasCanada, éléments d'une réflexion géographique (première et deuxième parties)», Cahiers de géographie du Québec, 24,62 (1980): 193-224, 24,63 (1980): 385-428. Citons enfin l'ouvrage de D. 
Dans toutes ces études l'agriculture est perçue à juste titre comme un maillon du rouage économique national ou international et la qualité du système agricole est évaluée en fonction de sa viabilité économique. C'est dire que jusqu'à présent, l'agriculture a été examinée sous une même et seule lentille, celle de l'économie.

Nous proposons ici d'aborder le sujet sous un angle différent, celui de l'écologie. Les anthropologues ont été les premiers à appliquer le corpus théorique de l'écologie à l'étude des systèmes agricoles préindustriels que l'on retrouve aujourd'hui encore dans certains pays en développement ${ }^{5}$. Le système agricole y est considéré comme un écosystème à la fois soumis aux lois universelles de la nature et à celles de la communauté à laquelle il appartient. La valeur du système s'exprime alors en termes d'efficacité et de viabilité écologiques ${ }^{6}$.

Appliquée à une société qui ne peut être appréhendée qu'à travers des vestiges documentaires, cette approche écologique ne peut donner sa pleine mesure. En effet, les documents sur lesquels se fonde notre étude ne contiennent pas tous les éléments nécessaires à la reconstitution des flux énergétiques ${ }^{7}$. Il nous manque par exemple la plupart des données sur la quantité d'énergie investie dans le système agricole. Il est toutefois possible de saisir, en termes peut-être plus qualitatifs, le réseau d'influence existant entre la stratégie agricole, l'environnement physique et le milieu social.

Dans cette perspective, nous comparerons ici les pratiques agricoles de Canadiens français et de Canadiens anglais (Irlandais, Écossais, Anglais ${ }^{8}$ dans deux milieux édaphiques différents (terres argi-

T. Ruddell, dans laquelle l'auteur analyse la situation des paysans de la région de Québec dans une perspective internationale, «Quebec City, 1765-1831: the Evolution of a Colonial Town» (Ottawa, Musée canadien des civilisations, 1987), chap. 2.

5 Citons par exemple l'étude de V. Smil sur la Chine rurale, «Energy Flow in Rural China», Human Ecology, 7 (1979): 119-131); celle de J. E. Ellis et al qui comparent les flux énergétiques de deux systèmes traditionnels d'élevage en Ouganda et aux Indes avec celui pratiqué en Amérique du Nord, «A Comparison of Energy Flow Among the Grazing Animals of Different Societies», Human Ecology, 7 (1980): 135-149; celle de F. Felice-Katz «Analyse éco-énergétique d'un élevage nomade Touareg», Annales de géographie, 89 (1980): 57-73; et enfin l'ouvrage de T. P. Bayliss-Smith, The Ecology of Agricultural systems (Cambridge, Cambridge University Press, 1982).

Ainsi un système très peu productif économiquement parlant, tel celui des Tsembaga de Nouvelle-Guinée, s'avère très efficace d'un point de vue écologique puisque pour chaque calorie investie il est capable de produire 14 calories de nourriture et que les méthodes de culture adoptées garantissent la pérennité du système. En revanche, le système agricole industriel des pays occidentaux engloutit entre cinq et dix calories de combustible fossile pour fournir une calorie alimentaire et connaît en outre de sérieux problèmes écologiques. Voir R. A. Rappaport, "The Flow of Energy in an Agricultural Society», Scientific American, 225 (1971): 117-132; J. de Rosnay, «Production agricole, un bilan énergétique qui se détériore», La Recherche, 47 (1974): 694-696.

7 Bayliss-Smith (1982) a réussi à utiliser une telle approche pour étudier un système agricole anglais du début du $19 \mathrm{e}$ siècle grâce à l'existence de descriptions détaillées d'un journaliste de l'époque.

${ }^{-}$Les immigrants anglais, irlandais et écossais que l'on désigne aujourd'hui sous le vocable de «Canadien anglais» constituent autant de groupes ethniques différents qui jouissaient de répu- 
leuses, terres sableuses). Notre tâche sera de chercher à comprendre de quelles façons les influences de l'environnement physique et du milieu social s'imbriquent et s'articulent entre elles pour orienter, voire déterminer la stratégie agricole. Loin de prétendre clore un débat qui alimente l'historiographie depuis plusieurs années, nous espérons par cette étude préciser certains contours de cette ébauche du paysan canadienfrançais.

\section{I - CADRE PHYSIQUE ET HUMAIN}

Par sa diversité ethnique et ses sols variés, le comté de Prescott fournit un site d'observation privilégié pour ce type d'étude comparative'. Situé à l'extrémité orientale de l'Ontario, il repose sur une assise de rocs sédimentaires à faible pendage qui confère au paysage son aspect tabulaire. En général, l'influence glaciaire est demeurée très discrète et le paysage a surtout été façonné par des processus postglaciaires. En effet, la mer Champlain déposa pendant deux ou trois mille ans d'importantes couches d'argile. Lorsqu'elle entama sa lente régression vers l'est, un nouveau réseau de drainage prit forme. L'Outaouais qui charriait des eaux chargées de sédiments forma un vaste delta à son embouchure dans la mer et les dépôts de ces sables deltaïques subsistent aujourd'hui ${ }^{10}$. Véritables îlots au milieu d'une mer d'argile, ils s'élèvent doucement à quelques mètres au-dessus de la plaine. Les sols qui ont évolué à partir de ces deux types de matériel parental sont dotés de propriétés physiques spécifiques et leur mise en valeur commandera, comme nous le verrons, des stratégies bien différenciées.

Entamé par les Loyalistes à la fin du $18 \mathrm{e}$ siècle, le peuplement de ce comté s'est poursuivi tout au long du siècle suivant alors que sont venus tour à tour se déverser les surplus des populations écossaise, irlandaise, anglaise et canadienne-française. Les Ecossais sont arrivés très tôt dans la région; ils se sont installés massivement dans le comté

\footnotetext{
tations très inégales en tant qu'agriculteurs. Les Écossais des «Highlands» et les Irlandais étaient en général sévèrement critiqués par les agronomes de l'époque tandis que les «Lowlanders» et les Anglais étaient considérés comme de bons agriculteurs, W. S. Reid, The Scottish Tradition in Canada (McClelland and Steward, 1976), 161-177. R. L. Jones, History of Agriculture in Ontario, 1613-1880 (Toronto, University of Toronto Press, 1977), 51. Nous avons par conséquent jugé préférable de traiter ces différentes ethnies séparément.

C. Gaffield a d'ailleurs utilisé ce territoire pour ses études comparatives sur les familles canadiennes-françaises et canadiennes-anglaises. C. Gaffield, «Canadian Families in Rural Context, Hypothesis from the Mid-Nineteenth Century» dans Communications historiques (Société historique du Canada, 1979), 48-70; «Boom and Bust: the Demography and Economy of the Lower Ottawa Valley in the Nineteenth Century» dans Communications historiques (Société historique du Canada, 1982), 172-196; «Schooling, the Economy, and Rural Society in Nineteenth-Century Ontario» dans J. Parr, Childwood and family in Canadian history (Toronto, McClelland \& Stewart, 1982), 69-92.

10 L. J. Chapman et D. F. Putnam, The Physiography of Southern Ontario (Toronto, University of Toronto Press, 1966).
} 
de Glengarry et ont très vite débordé dans les comtés voisins de Prescott et de Stormont. Quelque 2300 «Highlanders» originaires de la région d'Inverness ont ainsi gagné l'Est ontarien entre 1785 et $1815^{11}$. Puis les Irlandais ont commencé à affluer. Il s'agissait d'abord de tisserands durement touchés par la mécanisation; plus tard, entre 1846 et 1849 , la famine amena un nombre croissant de petits paysans pour qui le paiement des «poor rates» était devenu insupportable ${ }^{12}$. En 1851 les Irlandais de naissance comptaient pour 12 pour cent de la population du comté contre 7 pour cent pour les Écossais. Quant aux autres Britanniques, c'est-à-dire les Anglais et les Gallois, ils ne représentent qu'une faible proportion de la population et leur arrivée est peu documentée.

Dès le début du 19e siècle, les Bas-Canadiens des comtés de Vaudreuil et de Soulanges avaient peu à peu essaimé dans la région estontarienne mais ce n'est que vers les années 1840 que s'amorça la colonisation massive du comté de Prescott par les francophones ${ }^{13}$. Le peuplement francophone de cette région s'inscrit dans le mouvement de colonisation orchestré par le clergé et l'élite canadienne-française dès le milieu du 19e siècle en vue d'enrayer l'hémorragie démographique dont souffrait le Bas-Canada à cette époque.

Les avantages que présentait le comté de Prescott à la colonisation n'avaient pas échappé à l'évêque de Montréal, Mgr Bourget, qui souhaitait étendre son aire de juridiction au-delà de la frontière provinciale. Proche de la province de Québec, le comté offrait de vastes terres vacantes et permettait une colonisation en groupe. La proximité des chantiers assurait à la fois un débouché pour certains produits agricoles et une possibilité d'emplois saisonniers. Enfin, parce qu'il constituait en quelque sorte le prolongement de la région physiographique des basses terres du Saint-Laurent où s'était jusque-là confiné l'oecoumène des Canadiens français, ce territoire exerçait sur les colons un plus puissant pouvoir d'attraction que les terres du bouclier canadien sises de l'autre côté de l'Outaouais. Certains auteurs ${ }^{14}$ ont remarqué la préférence des Canadiens français pour les terres argileuses, lourdes et difficiles à travailler. Elles étaient dédaignées des Anglo-Saxons qui

11 M. McLean, «Achd and Rhigh: A Highland Response to the Assisted Emigration of 1815» dans D. H. Akenson, ed., Canadian Papers in Rural History, V (Gananoque, Langdale Press, 1986), 181-198. L. Brault, Histoire des comtés unis de Prescott et de Russell (L'Orignal, Conseil des comtés unis, 1965).

${ }_{12}$ D. H. Akenson, «Ontario: Whatever Happened to the Irish», D. H. Akenson, ed., Canadian papers in rural history, III (Gananoque, Langdale Press, 1980), 204-256. L. Brault, op. cit.

${ }_{13}$ La principale étude sur la colonisation francophone du comté de Prescott est celle de D. G. Cartwright, «Institutions on the Frontier: French Canadian Settlement in Eastern Ontario in the Nineteenth Century», Canadian Geographer, 21,1: 1-21. Voir également L. Brault, op. cit.

14 L. Brault, idem, 28; D. G. Cartwright, «Institutions on the Frontier»; C. Gaffield, «Boom and Bust», 185. 
choisissaient plutôt les terres hautes et les sols légers, issus de dépôts glaciaires ou deltaïques.

Le commerce du bois qui se développa au début du 19e siècle à la faveur des guerres napoléoniennes fut à l'origine du peuplement et de l'essor économique de la vallée de l'Outaouais. On y exploita tout d'abord le pin et le chêne équarris, fortement en demande dans les chantiers navals britanniques. Puis, à la mi-siècle, le commerce du bois ouvré avec les États-Unis s'implanta alors même que s'anémiait celui du bois équarri.

L'agriculture de la vallée de l'Outaouais profita des activités forestières de plusieurs façons: les chantiers offraient à la fois un débouché pour certains produits agricoles et un emploi saisonnier pour les hommes. Ces chantiers requéraient des quantités énormes de vivres et ce sont les fermiers de la vallée de l'Outaouais qui détenaient le monopole du commerce du foin et de l'avoine, denrées trop volumineuses pour être importées des États-Unis comme l'étaient la viande de porc et la farine. Ils écoulaient également des boeufs de trait, des chevaux ainsi que du beurre, des pois, des pommes de terre et du porc. Le prix des produits agricoles était plus élevé sur le marché de Bytown que partout ailleurs; aussi, même les habitants de Prescott les plus rapprochés de Montréal préféraient expédier leurs produits à Bytown plutôt que dans la métropole ${ }^{15}$.

Si la coupe forestière présentait des avantages certains pour l'agriculteur, il ne faut pas sous-estimer ceux que la colonisation apportait aux entrepreneurs. En effet, ils disposaient d'une source d'approvisionnement relativement proche et d'une main-d'oeuvre qualifiée et outillée. Cette relation symbiotique entre agriculture et exploitation forestière présente des similitudes avec le modèle de développement «agroforestier ${ }^{16}$ de certains fronts pionniers du Québec. Des différences majeures distinguent toutefois le comté de Prescott de ces régions dans la mesure où il s'agit ici d'un territoire qui, d'une part, bénéficie d'un climat et de sols propices à l'agriculture et qui, d'autre part, est relié à Montréal et à Ottawa par la rivière des Outaouais.

Mais dès le début des années 1870, les ressources forestières immédiatement accessibles manifestèrent des signes d'épuisement et les marchands de bois durent s'enfoncer plus profondément dans la forêt; ils s'approvisionnèrent graduellement dans les nouvelles fermes

15 R. L. Jones, History of Agriculture in Ontario, 1613-1880 (Toronto, University of Toronto Press, 1946), réimpression en 1977, 118.

${ }_{16}$ N. Séguin qui a étudié la région forestière du Saguenay, montre comment les intérêts forestiers ont assujetti l'agriculture, l'entraînant au-delà de sa zone climatique et à l'extérieur des circuits commerciaux et la maintinrent ainsi dans un état de subsistance, N. Séguin, La Conquête du sol au 19ième siècle (Québec, Boréal Express, 1977), 295 p. 
de la haute vallée de l'Outaouais ${ }^{17}$. L'agriculture du comté de Prescott et de la pointe est-ontarienne dut alors se réorienter; elle se dirigea lentement vers l'industrie laitière, activité qui, à cette époque, suscitait un intérêt grandissant. En 1870, année où se situe notre étude, le comté de Prescott compte déjà une population bien diversifiée. Les Canadiens français forment la moitié de la population, les Irlandais d'origine en représentent le quart, les Écossais environ 15 pour cent et les Anglais 7 pour cent. Du point de vue économique, les années 1870 se situent à la charnière de deux époques, l'une désormais vacillante du commerce avec les chantiers, et l'autre plus prometteuse de l'industrie laitière.

\section{II - APPROCHE ÉCOLOGIQUE ET MÉTHODE}

La relation que l'on veut établir entre stratégie agricole, environnement physique et milieu social exige que l'on définisse au préalable certains de ces termes.

La stratégie agricole peut se définir comme l'ensemble des actions déployées par le fermier pour extraire sa nourriture du sol soit directement par l'agriculture et/ou indirectement par l'élevage; elle implique donc une série de choix: choix des cultures, des assolements, des rotations, des animaux domestiques, des méthodes et des outils de travail ainsi que des sources d'énergie pour réaliser ce travail. Ces choix dépendent à la fois des contraintes environnementales auxquelles le fermier doit faire face et du milieu social et économique dans lequel il baigne.

Les contraintes environnementales sont multiples et peuvent être classées en deux catégories: celles qui influencent la croissance des plantes telles que la radiation solaire, la température, l'eau et les nutriments et celles qui affectent le travail de l'agriculteur comme, par exemple, la pierrosité, la pente du terrain ou la structure du sol. Puisque notre étude s'inscrit dans un espace relativement restreint, les limitations «atmosphériques» (radiation solaire, température et pluviométrie) sont analogues sur tout le territoire. Par contre, la facilité d'un sol à être travaillé, ses capacités de rétention d'eau et sa teneur en éléments nutritifs varient en fonction de sa qualité de sorte que c'est le «type de sol» qui constituera le facteur environnemental essentiel pour cette étude.

Une application rigoureuse de l'approche écologique suppose que nous puissions minimiser l'effet discriminant de certains facteurs, tels l'accès au marché, l'ancienneté de la ferme ou le mode de faire-valoir.

17 C. Gaffield, «Schooling, the Economy, and Rural Society in Nineteenth-Century Ontario», 74; W. E. Greening, «The Lumber Industry in the Ottawa Valley and the American Market in the 19th Century», Ontario History, 62: 134-136. 
TABLEAU 1

Répartition des fermes, comté de Prescott, Ontario, 1871

\begin{tabular}{|lccc|}
\hline \multicolumn{1}{|c}{$\begin{array}{c}\text { Appartenance } \\
\text { ethnique }\end{array}$} & $\begin{array}{c}\text { Terres } \\
\text { argileuses }\end{array}$ & $\begin{array}{c}\text { Terres } \\
\text { sableuses }\end{array}$ & Total \\
\hline Canadiens français & 15 & 9 & 24 \\
Irlandais & 7 & 10 & 17 \\
Écossais & 7 & 6 & 13 \\
Anglais & 5 & 9 & 14 \\
TOTAL & 34 & 34 & 68 \\
\hline
\end{tabular}

Source: Recensement de 1871.

Pour ce faire, nous avons d'abord restreint le territoire d'étude au secteur des cantons de Plantagenet nord et sud compris entre les $7 \mathrm{e}$ et $20 \mathrm{e}$ concessions. Ce périmètre offre deux principaux avantages: 1) les cultivateurs y ont une chance à peu près égale d'accéder au marché puisque les lots choisis se situent tous à une faible distance de la rivière Nation Sud ou de son affluent navigable, la rivière Scotch;2) la superficie des plaines d'argile, mais surtout des régions sableuses, est suffisamment étendue pour pouvoir contenir intégralement un nombre convenable d'exploitations agricoles (figure 1). Nous avons ensuite éliminé systématiquement toute ferme ne possédant pas au moins 25 acres défrichés et n'ayant pas constitué une exploitation agricole en 1861, ce qui nous a assuré de ne conserver que des fermes relativement bien établies. Les quelques fermes dont l'occupant n'était pas propriétaire ont également été écartées ${ }^{18}$. Enfin nous avons rejeté celles dont le propriétaire exerçait un autre métier comme celui de forgeron ou de marchand, par exemple.

Ceci fait, nous avons procédé à la localisation des exploitations sur une carte de cadastre puis sur une carte des sols. Tous les lots se trouvant à cheval entre deux types de sol ont, pour des raisons bien évidentes, été éliminés. Nous avons ainsi obtenu un groupe de 68 fermes dont la répartition apparaît à la figure 2 et au tableau 1. Les recensements nominatifs constituent la principale source d'informations pour cette étude. Ils permettent non seulement d'obtenir des informations

18 En 1871, le comté de Prescott compte 91 pour cent de cultivateurs propriétaires de leur exploitation. Canada, Recensement du Canada 1870-1871, vol. 3, Tableau XXI. 
FIGURE 1

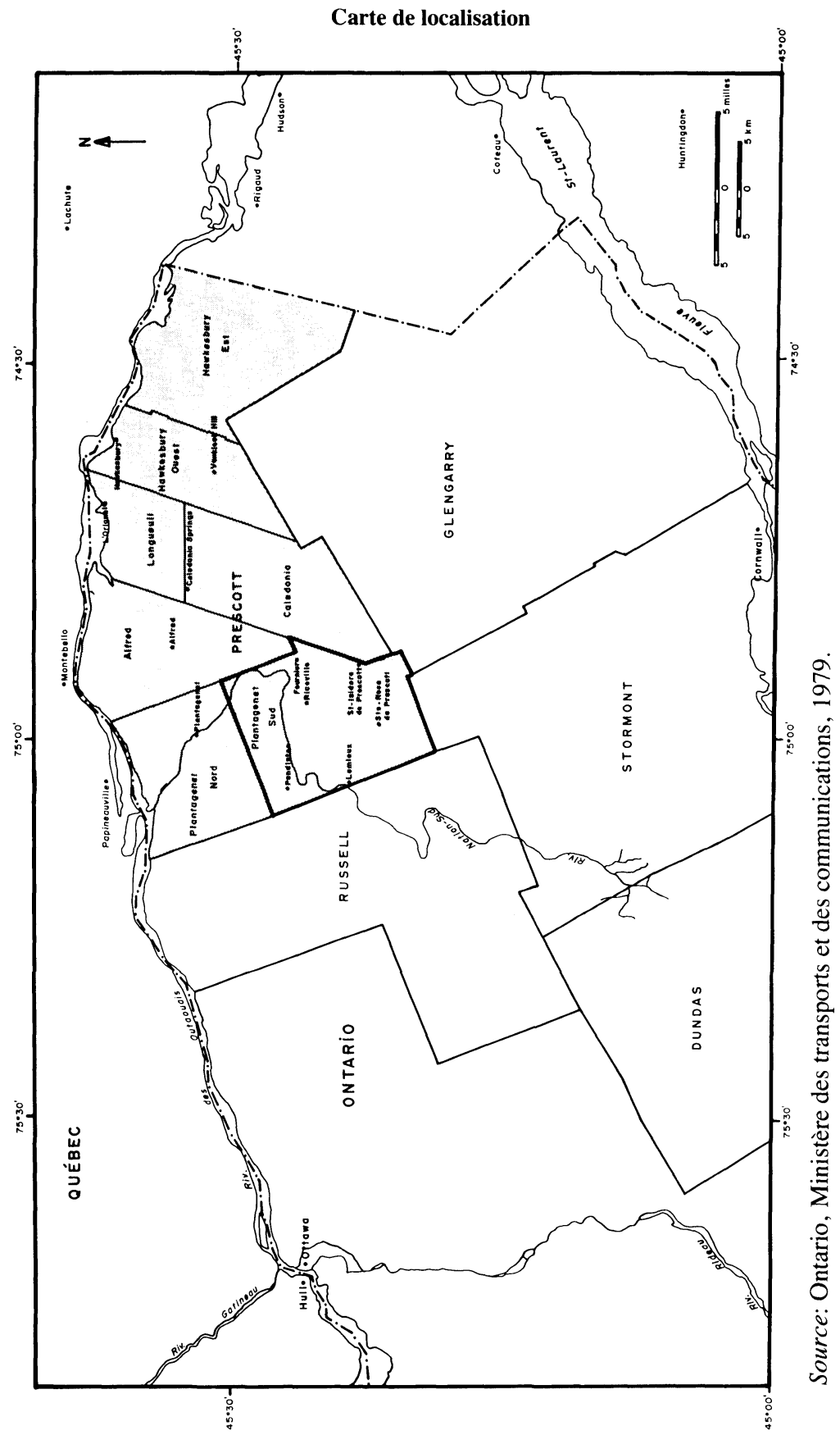


FIGURE 2

Localisation des fermes

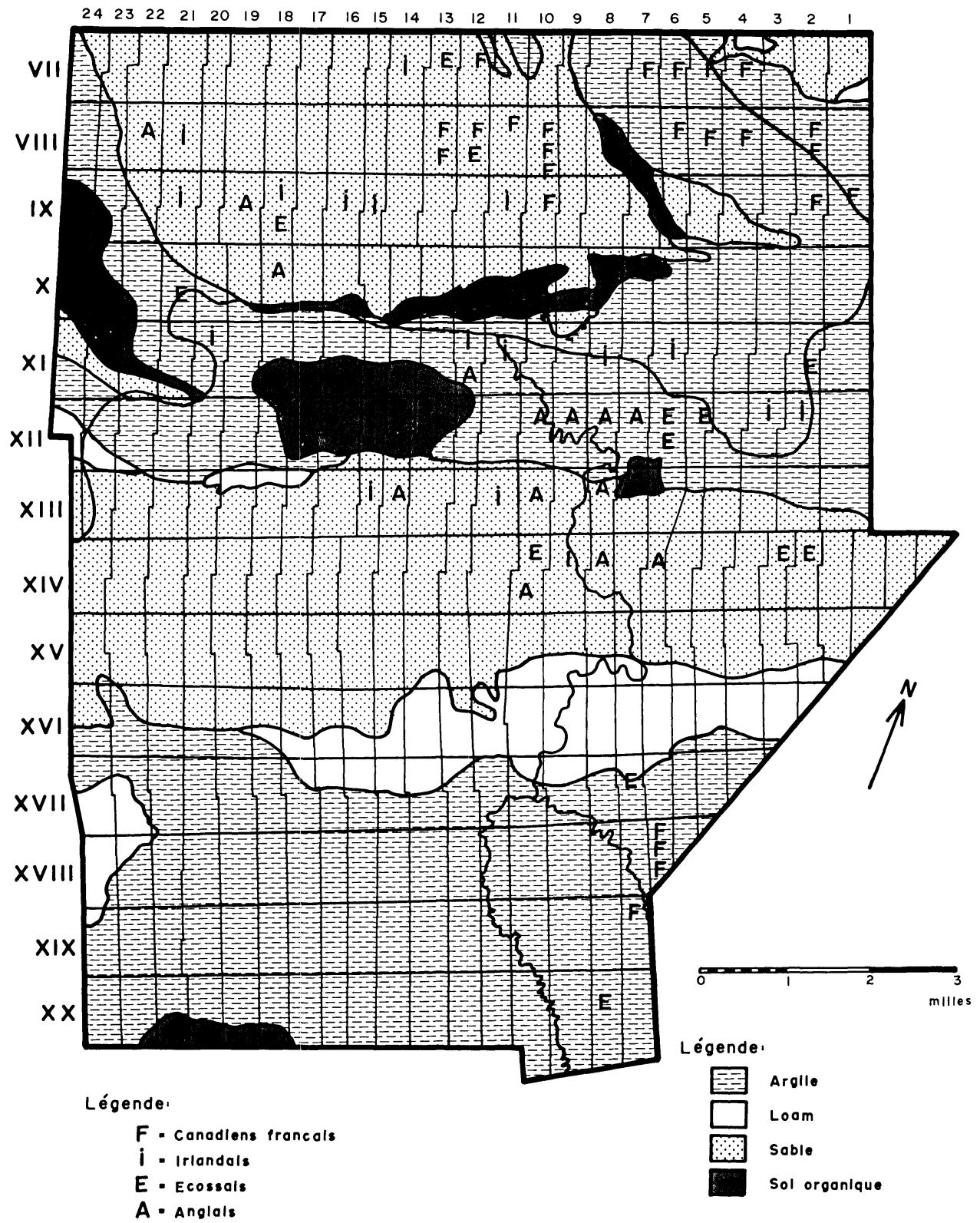

Source: Ontario, Ministère des Transports et des Communications, 1979. Richards et Wicklund, 1962. 
précises sur chaque famille et sur certaines de leurs pratiques agricoles mais également de situer chacune des propriétés sur une carte de cadastre et sur une carte des sols ${ }^{19}$. Dans le comté à l'étude, ces recensements n'ont subsisté que pour les années 1861 et 1871. Comme les Canadiens français ont colonisé cette région à une période relativement tardive (à partir des années 1840), nous avons préféré celui de 1871 qui correspondait à l'époque où bon nombre d'entre eux étaient déjà bien établis.

Deux principales méthodes s'offrent à nous pour étudier la stratégie agricole de ces différents groupes ethniques. La première est de grouper les fermes en fonction de l'appartenance ethnique et du type de sol et de comparer pour chacun des groupes la valeur moyenne des variables agricoles (rendements, volumes des récoltes, cheptel etc.). Cette méthode présente deux principaux inconvénients: 1) d'abord une perte d'information, inhérente à tout calcul de moyenne, sur le caractère individuel de chaque ferme, 2) ensuite une difficulté de manipulation sinon d'interprétation reliée au grand nombre de variables et de groupes.

La seconde méthode, qui est apparue plus appropriée, procède à l'inverse de la première. Elle consiste à établir une classification typologique des fermes en fonction de caractéristiques purement agricoles (rendements, volumes des récoltes, cheptel, etc.) et de rechercher, en un deuxième temps, les liens existant entre ces groupes de fermes et les facteurs ethnique et édaphique. La classification typologique a été effectuée à l'aide de méthodes multi-variées informatisées ${ }^{20}$; elle comporte trois principales étapes:

1) Nous avons d'abord sélectionné parmi les données du recensement agricole 23 variables qui décrivent quatre aspects de la stratégie agricole:

- l'utilisation du sol (surface défrichée, surfaces réservées aux pâturages, au foin, aux pommes de terre, au blé)

- le rendement à l'acre (du blé, du foin et de la pomme de terre, soit les seuls rendements disponibles dans ce recensement)

19 Ils peuvent toutefois comporter un certain nombre d'embûches dont les risques d'erreurs liés à l'utilisation de systèmes de mesure différents par les francophones et les anglophones du Québec. En Ontario, où seul le système britannique est en vigueur, on peut considérer que de tels risques sont minimes. Il est peu probable que l'on ait persisté à utiliser des mesures de surface françaises dans des cantons divisés en acres. Pour ce qui est des mesures de volume, les recenseurs restent muets sur le sujet et rien ne laisse supposer que les minots aient été d'usage courant dans la région. Les chances que certains Canadiens français aient évalué leurs récoltes en minots sans que le recenseur ne l'ait signalé sont faibles puisqu'en 1871 le problème était bien connu. Disons enfin que si la chose s'était produite dans quelques cas, cela ne saurait changer nos résultats de façon significative. (N. Fortier, «Les recensements canadiens et l'étude de l'agriculture québécoise, 1851-1901», Histoire sociale, 17,34: 257-287; R. M. McInnis, «Some Pitfalls in the 18511852 Census of Agriculture of Lower Canada», Histoire sociale, 14,27 (1981): 219-231; N. Séguin, «L'agriculture de la Mauricie et du Québec», RHAF, 35,4 (mars 1982): 537-562).

20 Pour une description détaillée de ces méthodes d'analyse multivariée nous renvoyons le lecteur au premier chapitre de la thèse, 17 . Nous avons utilisé le programme SPSS pour l'analyse en composantes principales et le programme BMDP-2M pour l'analyse de groupement. 
- le cheptel (le nombre de vaches laitières, de chevaux, de porcs et de moutons)

- le volume des récoltes (le blé, l'orge, l'avoine, le seigle, les pois, le sarrasin, le maïs, la pomme de terre et le foin; nous avons omis les cultures peu ou pas pratiquées telles celle des graines de lin, des racines, des fruits, du houblon dont l'importance est secondaire pour la caractérisation de la stratégie agricole $)^{21}$.

2) Une analyse en composantes principales a ensuite été effectuée sur ces 23 variables afin de définir des critères de groupement ${ }^{22}$. Cette analyse a essentiellement pour but de substituer à l'ensemble de variables initiales un nombre plus réduit de variables appelées «composantes», cette économie étant réalisée avec une perte minimum d'information. Ces composantes constituent les critères synthétiques sur lesquels se fondera la classification.

3) Enfin l'analyse de groupement consiste à mesurer le degré de similarité entre les différentes fermes pour l'ensemble des composantes et de constituer des groupes de fermes en fonction de leur ressemblance.

Nous avons ainsi obtenu quatre principaux groupes réunissant 44 fermes et 3 groupes mineurs ne totalisant que 7 fermes; enfin 15 fermes sont demeurées non groupées en raison de la singularité de leurs pratiques agricoles. Dans le cadre de cet article nous restreindrons notre propos à l'étude des quatre principaux groupes qui représentent à eux seuls 65 pour cent des exploitations originales.

\section{III - GROUPES DE FERMES, APPARTENANCE ETHNIQUE ET TYPE DE SOL}

Une classification typologique des fermes vient d'être définie en fonction de ce qu'on y récolte, de ce qu'on y élève et de la manière dont on occupe effectivement le sol. Cherchons maintenant à voir s'il existe une correspondance entre ces quatre principaux groupes de fermes et les facteurs ethnique et édaphique qui ont été jusqu'à présent volontairement tenus à l'écart.

\footnotetext{
21 Sur les 68 exploitations à l'étude, 2 seulement produisent des navets, 3 des fruits (moins de 4 boisseaux), 4 récoltent des quantités minimes d'autres racines, tandis que 3 autres recueillent 1 boisseau de lin chacune. Quant au houblon qui deviendra une des principales cultures de rente de la région quelques décennies plus tard, il est totalement absent en 1870.

${ }_{22}$ Ces données ont été pondérées afin de contrer un effet «taille» indésirable qui fausserait la signification des coefficients de corrélation. Les valeurs sont exprimées non pas en chiffre absolu mais relativement à la surface améliorée.
} 
Le tableau 2 et la figure 3 montrent la correspondance non équivoque qui existe entre le type de sol et les groupes de fermes. En effet, les fermes des groupes $\mathrm{A}$ et $\mathrm{D}$ sont exclusivement cantonnées sur les terres argileuses tandis que celles du groupe $\mathrm{B}$ et $\mathrm{C}$ se rencontrent majoritairement sur les sols sableux.

Sans être aussi tranché, le lien entre l'appartenance ethnique du chef de famille et les groupes de fermes est évident. Le tableau 3 révèle que le groupe A est majoritairement anglo-saxon et que les Anglais et les Irlandais représentent $70 \%$ du groupe B. Toutefois, la présence des Canadiens français dans ce dernier groupe est appréciable puisqu'elle équivaut au tiers du nombre total de fermes canadiennes-françaises sur les sols sableux. Par contre, les Écossais y sont faiblement représentés. Le groupe $\mathrm{C}$ réunit une majorité (70\%) de Canadiens français et d'Écossais. Enfin l'identité ethnique du dernier groupe ne laisse aucun doute: tous les membres, à une exception près, sont canadiens-français.

Le critère de Kullback ${ }^{23}$ a été calculé afin d'évaluer le degré de signification de ces résultats. Les correspondances entre les facteurs ethnique et édaphique et les groupes de fermes se sont avérées hautement significatives: les chances que les liaisons observées entre le type de sol et les groupes soient le fruit du hasard sont de 5 sur 1000 alors qu'elles se situent autour de 3 sur 100 dans le cas de l'appartenance ethnique.

TABLEAU 2

Relation entre groupes de fermes et type de sol, comté de Prescott, Ontario, 1871

\begin{tabular}{|cccccc|}
\hline $\begin{array}{c}\text { Groupe de } \\
\text { fermes }\end{array}$ & $\begin{array}{c}\text { N total } \\
\text { de fermes }\end{array}$ & \multicolumn{2}{c|}{ SOLS ARGILEUX } & \multicolumn{2}{c|}{ SOLS SABLEUX } \\
\hline A de fermes & $\%$ & N de fermes & $\%$ \\
B & 7 & 7 & $100 \%$ & - & - \\
C & 13 & 1 & $8 \%$ & 12 & $92 \%$ \\
D & 16 & 5 & $31 \%$ & 11 & $69 \%$ \\
\hline
\end{tabular}

Source: Recensement de 1871.

${ }^{23}$ Le lecteur désireux de connaître les détails des calculs pourra consulter notre thèse aux pages 79 à 81 . Ce test de Kullback est utilisé dans les cas comme celui-ci, où la faiblesse des effectifs à l'intérieur de chaque sous-groupe, interdit l'utilisation du $\mathrm{X}^{2}$. 
FIGURE 3

Répartition des groupes de fermes sur les différents types de sol

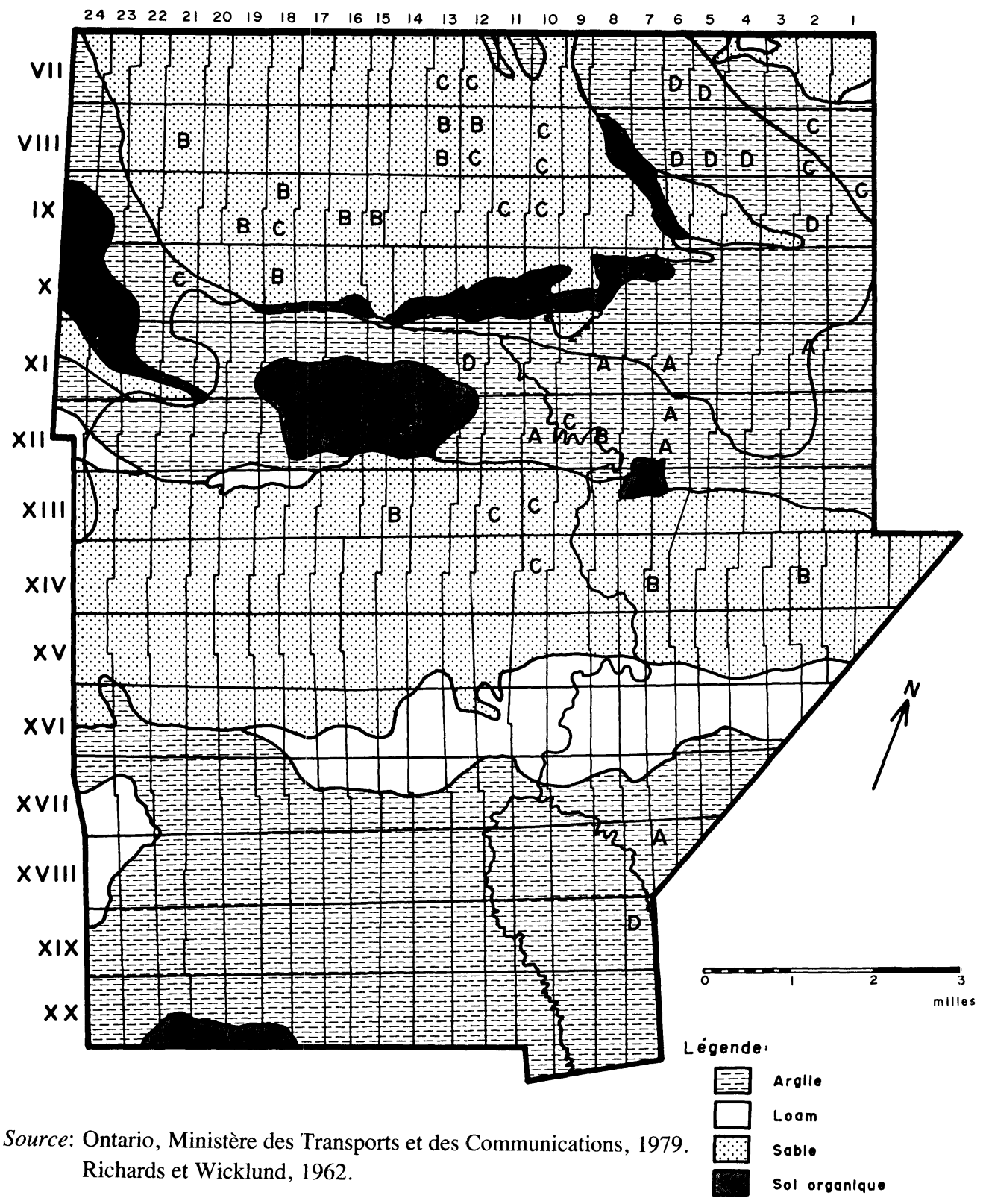


TABLEAU 3

Relation entre groupes de fermes et appartenance ethnique, comté de Prescott, Ontario, 1871

\begin{tabular}{|cccccc|}
\hline $\begin{array}{c}\text { Groupe de } \\
\text { fermes }\end{array}$ & $\begin{array}{c}\text { N de } \\
\text { fermes }\end{array}$ & $\begin{array}{c}\text { Canadiens } \\
\text { français }\end{array}$ & Irlandais & Écossais & Anglais \\
\hline A & 7 & 1 & 2 & 3 & 1 \\
B & 13 & 3 & 4 & 1 & 5 \\
C & 16 & 7 & 2 & 5 & $\frac{2}{8}$ \\
D & $\frac{8}{44}$ & $\frac{7}{18}$ & $\frac{1}{9}$ & $\overline{7}$ & $\overline{7}$ \\
\hline
\end{tabular}

Source: Recensement de 1871.

Ces quatre groupes de fermes qui n'étaient au départ que des entités purement agronomiques sont maintenant revêtus d'une signification écologique et ethnique. Il apparaît clairement que les quatre groupes représentent deux types d'adaptation écologique distincts dans chacun des deux types de conditions édaphiques et que chacun de ces comportements semble être adopté de préférence par des groupes ethniques particuliers. Mais notre propos est bien moins de confirmer des influences dont nous soupçonnions fortement l'existence que de découvrir de quelles façons elles s'exercent. Pour ce faire il nous faut voir de plus près ce qui caractérise chacun de ces groupes.

Le groupe A réunit des anglophones dont les fermes sont toutes situées sur des terres argileuses. Ces cultivateurs pratiquent une agriculture intensive et 85 pour cent de la surface améliorée est ensemencée. Le pourcentage de terre réservé aux pâturages est particulièrement bas. Parmi les cultures, le blé tient une place importante, recouvrant 14 pour cent des terres défrichées; les pommes de terre en revanche, n'en occupent qu'un pour cent. Les tableaux des rendements (tableau 5) et des récoltes (tableau 6) indiquent que ces fermes sont hautement productives comparativement à celles des autres groupes; observons par exemple les rendements de blé et de foin ainsi que le volume des récoltes de pois, d'orge, d'avoine et de maiis. Quant au cheptel, le tableau 7 indique clairement qu'on trouve dans le groupe A le plus grand nombre de vaches laitières ainsi qu'un nombre relativement élevé de cochons ${ }^{24}$.

Dans le groupe B, nous retrouvons une majorité d'Irlandais et d'Anglais cultivant presque exclusivement des sols sableux. L'utilisa-

${ }^{24}$ En 1871, le nombre moyen d'animaux par ferme en Ontario et au Québec est respectivement de 3,7 et 3,4 dans le cas des vaches laitières, de 5,0 et de 3,1 dans le cas des porcs et de 8,8 et 8,5 dans le cas des moutons. 
TABLEAU 4

Utilisation du sol, comté de Prescott, Ontario, 1871

\begin{tabular}{|c|ccc|c|c|}
\hline \multirow{2}{*}{$\begin{array}{c}\text { Groupes de } \\
\text { fermes }\end{array}$} & Foin + Pâturage $=$ Herbe & Blé & $\begin{array}{c}\text { Pommes de } \\
\text { terre }\end{array}$ \\
\cline { 2 - 5 } & & & & & 1,0 \\
\hline A & 11,8 & 4,0 & 15,8 & 14,1 & 5,0 \\
B & 18,9 & 29,2 & 48,5 & 5,7 & 3,6 \\
C & 10,9 & 10,3 & 21,3 & 6,6 & 1,4 \\
D & 25,1 & 10,0 & 35,2 & 16,3 & \\
\hline
\end{tabular}

Source: Recensement de 1871.

TABLEAU 5

Rendements moyens par ferme ${ }^{\mathrm{a}}$, comté de Prescott, Ontario, 1871

\begin{tabular}{|lcccc|}
\hline Cultures & A & B & C & D \\
\hline $\begin{array}{l}\text { Blé } \\
\quad \text { (bois./acre) }\end{array}$ & 15,3 & 12,3 & 4,9 & 9,4 \\
$\begin{array}{l}\text { Foin } \\
\quad \text { (ton./acre) }\end{array}$ & 1,5 & 0,9 & 0,9 & 0,7 \\
$\begin{array}{l}\text { Pommes de terre } \\
\text { (bois./acre) }\end{array}$ & 65,5 & 102,1 & 58,8 & 39,6 \\
$\begin{array}{l}\text { a Les rendements moyens ont été calculés à partir des seules fermes pratiquant la } \\
\text { culture en question. }\end{array}$ & & & & \\
\hline
\end{tabular}

Source: Recensement de 1871.

tion du sol est extensive avec près de 50 pour cent de la surface améliorée réservée aux fourrages (comprenant foin et pâturages). C'est ici que l'on entretient les plus vastes pâturages (tableau 4). La moitié seulement des terres cultivables est ensemencée. L'espace affecté à la culture de la pomme de terre est relativement élevé alors qu'au contraire celui destiné au blé est plutôt faible. Les rendements sont un peu moins élevés que ceux du groupe précédent dans le cas du blé et du foin mais ils s'avèrent remarquables dans celui des pommes de terre (tableau 5). Le volume important de certaines récoltes telles l'avoine, le foin et les pommes de terre, laisse croire qu'il s'agit de fermes relativement productives (tableau 6). Les animaux et en particulier les moutons y sont très bien représentés (tableau 7). 
Le groupe $\mathrm{C}$ compte une majorité de cultivateurs canadiens-français et écossais dont les propriétés se trouvent principalement sur des sols sableux. Ils consacrent une portion de terre relativement restreinte à la production de fourrages. Les rendements de blé et de pommes de terre sont nettement inférieurs à ceux des deux groupes précédents. Dans l'ensemble, le volume des récoltes apparait faible, particulièrement celui du blé, des pois, du foin, de l'orge et de l'avoine (tableau 6 ). Seule la production de seigle et de sarrasin s'élève au-dessus de celle des autres groupes. Le nombre d'animaux est dans tous les cas le plus faible de tous (tableau 7).

\section{TABLEAU 6}

Récoltes moyennes par ferme (en boisseaux), comté de Prescott, Ontario, 1871

\begin{tabular}{|l|rrrr|}
\hline Cultures & \multicolumn{1}{|c}{ A } & \multicolumn{1}{c|}{ B } & \multicolumn{1}{c|}{ C } & D \\
\hline Blé & 101,0 & 36,5 & 9,3 & 67,5 \\
Pommes de terre & 32,3 & 243,8 & 76,9 & 16,5 \\
Pois & 48,4 & 21,8 & 8,8 & 38,8 \\
Foin (tonnes) & 9,0 & 11,1 & 3,1 & 7,9 \\
Orge & 57,4 & 3,5 & 2,0 & 7,5 \\
Avoine & 268,9 & 297,7 & 139,1 & 195,6 \\
Seigle & - & - & 2,2 & - \\
Sarrasin & 1,4 & 3,1 & 3,3 & 0,7 \\
Maïs & 6,2 & 7,7 & 3,9 & 0,6 \\
\hline
\end{tabular}

Source: Recensement de 1871.

TABLEAU 7

Cheptel,

comté de Prescott, Ontario, 1871

\begin{tabular}{|c|c|c|c|c|c|}
\hline \multirow[b]{2}{*}{$\begin{array}{l}\text { Groupes de } \\
\text { fermes }\end{array}$} & \multicolumn{4}{|c|}{ Nombre de bêtes par ferme } & \multirow[b]{2}{*}{$\begin{array}{c}\mathrm{N} \text { de chevaux par } \\
100 \text { acres de } \\
\text { terre labourée }\end{array}$} \\
\hline & $\begin{array}{l}\text { Vaches } \\
\text { laitières }\end{array}$ & Moutons & Cochons & Chevaux & \\
\hline A & 5,6 & 5,7 & 4,7 & 2,4 & 6,0 \\
\hline B & 4,6 & 9,7 & 4,8 & 2,3 & 8,2 \\
\hline $\mathrm{C}$ & 2,8 & 2,8 & 2,9 & 1,7 & 7,2 \\
\hline D & 2,8 & 7,3 & 4,5 & 2,3 & 8,1 \\
\hline
\end{tabular}

Source: Recensement de 1871. 
Dans le groupe D se retrouvent des Canadiens français cultivant essentiellement des terres argileuses. Contrairement aux anglophones du groupe A situés sur le même type de sol, les Canadiens français du groupe D pratiquent un type d'agriculture plus extensif: on remarque que le foin occupe le quart de l'espace amélioré. Les soles de blé recouvrent une forte proportion de l'espace ensemencé (tableau 4). Les rendements apparaissent généralement bas, particulièrement dans le cas du foin et des pommes de terre (tableau 5) et le volume des récoltes est plutôt faible à exclusion du blé et des pois (tableau 6). Quant aux animaux, leur nombre est relativement élevé sauf dans le cas des vaches laitières (tableau 7).

Ce portrait sommaire des différents groupes nous permet d'ores et déjà de percevoir certaines tendances. La première est que les Canadiens français, lorsque situés sur des terres argileuses, semblent favoriser une stratégie qui leur est propre et qui se distingue de celle des anglophones par une forme d'utilisation du sol plus extensive, des rendements moins élevés et une production plus faible. Sur les terres sableuses au contraire, le comportement des Canadiens français se confond avec celui des anglophones: on les retrouve associés aux Anglais et aux Irlandais du groupe B avec lesquels ils pratiquent manifestement une agriculture florissante, mais surtout associés aux Écossais du groupe C qui n'obtiennent que de faibles rendements.

Une seconde constatation s'impose, c'est que les Canadiens français se retrouvent en majorité dans les deux groupes (C et D) qui affichent les plus faibles productivités.

\section{IV - LES STRATÉGIES AGRICOLES}

The incoming of French Canadians introduced nothing new into the agriculture of Eastern Ontario. At worst they clung to the traditional practices of the St-Lawrence valley and at best, they imitated as well as they could what they saw of the farming of the remaining English-speaking inhabitants. ${ }^{25}$

Les résultats de notre analyse viendraient-ils corroborer les propos quelque peu méprisants de Jones? Comment expliquer la sur-représentation des Canadiens français dans les deux groupes $(C$ et $D)$ où l'on observe les plus faibles rendements, les récoltes les moins abondantes et un cheptel souvent plus pauvre? On pourrait certes répéter les arguments déjà avancés et plaider l'ignorance des principes agronomiques ou encore invoquer l'inertie naturelle, la mauvaise volonté, l'entête-

\footnotetext{
25 R. L. Jones, History of agriculture, 305. Malgré le fait que les travaux de Jones datent et que ses interprétations concernant les Canadiens français soient controversées, il est le seul historien de l'agriculture à s'être penché sur le comté de Prescott.
} 
ment d'un peuple à utiliser des méthodes vétustes. Mais ces interprétations laissent une trop large part à la subjectivité. Considérant la stratégie agricole comme le produit de l'interaction entre le milieu social et l'environnement physique, nous préférons évaluer cette stratégie à la lumière des moyens physiques et humains dont disposait le paysan. Les recensements fournissent à cet égard de précieux renseignements sur la famille et le niveau de richesse, renseignements susceptibles de fournir un éclairage nouveau sur la situation des cultivateurs de ces deux groupes à faible productivité et par le fait même sur celle des Canadiens français qui s'y trouvent en grand nombre.

\section{1 - Les caractéristiques de la famille}

La taille moyenne des familles en chiffre absolu a une signification écologique limitée en elle-même puisque la stratégie adoptée dépend également de la superficie de terre disponible. Nous avons donc calculé le nombre de personnes par unité de surface améliorée. Le tableau 8 montre que la densité de population est supérieure dans les deux groupes les moins productifs ( $\mathrm{C}$ et $\mathrm{D})$. Une analyse effectuée pour la totalité des 68 fermes composant l'échantillon révèle que le nombre de personnes par famille dépend beaucoup de l'origine ethnique. Les Canadiens français constituent les familles les plus populeuses avec une moyenne de 7,6 personnes; ils sont suivis de près par les Anglais $(7,4)$ et les Irlandais $(7,0)$. La taille de la famille écossaise se situe en revanche autour de 5,6 personnes.

\section{TABLEAU 8}

Caractéristiques des familles, comté de Prescott, Ontario, 1871

\begin{tabular}{|c|c|c|c|}
\hline \multirow{2}{*}{$\begin{array}{l}\text { Groupes de } \\
\text { fermes }\end{array}$} & \multicolumn{2}{|c|}{ Nombre de personnes par } & \multirow{2}{*}{$\begin{array}{l}\text { Âge du chef } \\
\text { de famille }\end{array}$} \\
\hline & Famille & 100 acres améliorés & \\
\hline $\mathrm{A}$ & 5,9 & 12,1 & 48,7 \\
\hline B & 8,1 & 13,8 & 50,9 \\
\hline C & 6,5 & 17,8 & 41,9 \\
\hline D & 6,8 & 15,2 & 39,6 \\
\hline
\end{tabular}

Source: Recensement de 1871.

Lorsqu'on examine l'âge du chef de famille, on se rend compte que les groupes à hauts rendements (A et B) correspondent aux familles les plus vieilles où l'âge du père varie entre 49 et 51 ans. Dans les deux autres groupes ( $\mathrm{C}$ et $\mathrm{D})$, la moyenne d'âge du père de famille ne dépasse pas 42 ans. Cette nette démarcation est significative puisqu'on peut 
supposer qu'une famille plus âgée a eu la possibilité d'accumuler un certain capital lui permettant, par exemple, de s'outiller et de diminuer d'autant la quantité de travail. D'autre part ces familles peuvent bénéficier de la force de travail supplémentaire des enfants non encore établis à leur propre compte. Ici encore, la moyenne d'âge du père de famille a un lien direct avec l'origine ethnique. En effet, dans l'échantillon en général, les chefs de ménage canadiens-français comptent parmi les plus jeunes avec une moyenne d'âge de 43 ans tandis que les pères de familles écossais atteignent 52 ans en moyenne. La jeunesse des familles canadiennes-françaises pourrait s'expliquer par le fait que ceuxci aient colonisé le comté à une période relativement récente et que les très jeunes couples aient été les plus enclins à émigrer.

La force de travail comprend celle des hommes et des femmes âgés de 14 à 65 ans; nous négligerons donc ici la contribution des enfants et des vieillards ${ }^{26}$. D'après le tableau 9 la force de travail est significa-

\section{TABLEAU 9}

Main-d'oeuvre active, comté de Prescott, Ontario, 1871

\begin{tabular}{|c|c|c|c|c|}
\hline \multirow{2}{*}{$\begin{array}{l}\text { Groupes de } \\
\text { fermes }\end{array}$} & \multicolumn{2}{|c|}{$\begin{array}{l}\text { Population active } \\
\text { par famille }\end{array}$} & \multicolumn{2}{|c|}{$\begin{array}{c}\text { Population active } \\
\text { par } 100 \text { acres améliorés }\end{array}$} \\
\hline & Hommes & Femmes & Hommes & Femmes \\
\hline A & 2,3 & 1,7 & 4,7 & 3,5 \\
\hline B & 2,9 & 2,7 & 4,9 & 4,6 \\
\hline C & 1,5 & 1,5 & 4,1 & 4,1 \\
\hline D & 1,6 & 1,1 & 3,6 & 2,5 \\
\hline
\end{tabular}

Source: Recensement de 1871.

tivement moins abondante dans les deux groupes à faible productivité (C et D) et ce, qu'on la considère en chiffre absolu ou en relation avec la taille de la ferme; la seule exception étant la densité élevée des effectifs féminins dans le groupe $\mathrm{C}$.

\section{2 - Le niveau de richesse}

Parmi les différents indices disponibles pour évaluer le niveau de richesse, retenons la taille des terres et la variété des instruments aratoires. Tel que l'indique le tableau 10 , les groupes $\mathrm{C}$ et $\mathrm{D}$ détiennent

\footnotetext{
${ }^{26}$ La fréquentation scolaire étant légèrement différente chez les Canadiens français et chez les Canadiens anglais dans le comté de Prescott, nous avons exclu de la main-d'oeuvre active les rares adolescents encore inscrits à l'école. Voir l'étude de C. Gaffield, «Canadian Families in Rural Context», 48-70.
} 
TABLEAU 10

Taille des terres, comté de Prescott, Ontario, 1871

\begin{tabular}{|c|ccc|}
\hline $\begin{array}{c}\text { Groupes de } \\
\text { fermes }\end{array}$ & $\begin{array}{c}\text { Nombre d'acres } \\
\text { occupés }\end{array}$ & $\begin{array}{c}\text { Nombre d'acres } \\
\text { améliorés }\end{array}$ & $\begin{array}{c}\text { \% d'acres } \\
\text { améliorés }\end{array}$ \\
\hline A & 125,0 & 48,7 & 42,9 \\
B & 105,8 & 58,8 & 63,2 \\
C & 90,6 & 36,5 & 44,7 \\
D & 69,4 & 44,7 & 69,2 \\
\hline
\end{tabular}

Source: Recensement de 1871.

une surface occupée ${ }^{27}$ très inférieure à celle des deux autres groupes. On remarque toutefois que les cultivateurs du groupe $\mathrm{D}$ ont défriché une forte proportion de leurs terres de sorte que la surface améliorée est à peine plus réduite que celle du groupe $\mathrm{A}$. Le groupe $\mathrm{C}$, au contraire, dispose d'un espace amélioré très restreint. Une fois de plus, les fermes des groupes $\mathrm{C}$ et $\mathrm{D}$ à faible productivité sont les moins bien pourvues.

Dans l'échantillon en général, on remarque que la surface occupée atteint à peine 80 acres chez les Canadiens français alors qu'elle s'élève à environ 115 chez les Irlandais et les Écossais et à 120 chez les Anglais. Quand au nombre d'acres améliorés, les Canadiens français se retrouvent aussi au bas de l'échelle avec 42 acres tandis que les Irlandais et les Anglais disposent d'une moyenne de 55 acres.

Les instruments aratoires sont déterminants dans le choix de la stratégie agricole puisqu'ils affectent directement le travail de l'agriculteur et par conséquent la quantité d'énergie investie dans le système. Comme le fait remarquer Irwin:

Farmers must do everything in season since production depends on timing. Yet the amount of work that can be done in any one season rests heavily on the energy available to the farmer. Implements, or tools, are essential instruments through which energy is converted to work. ${ }^{28}$

Les charrues, les herses et les cultivateurs ${ }^{29}$, regroupés sous la même rubrique dans le recensement, sont les instruments les plus répandus. La plupart des 68 paysans à l'étude possèdent au moins deux de ces

\footnotetext{
27 Dans le recensement de 1871, la superficie de l'exploitation agricole, c'est-à-dire l'ensemble des surfaces améliorées et non améliorées, est désignée par le vocable «terres occupées».

${ }_{28}$ R. W. Irwin, «Energy, from Man to Machine», T. A. Crowley, First Annual Agricultural History of Ontario Seminar Proceedings (Guelph, 1976), 39. terre.
} 
pièces, probablement les indispensables charrues et herses ${ }^{30}$. Comme la moyenne de deux instruments n'est pas atteinte dans les groupes $\mathrm{C}$ et $\mathrm{D}$, on peut supposer que tous ont une charrue mais que certains n'ont pas de herse. Dans les deux autres groupes, la moyenne supérieure à deux instruments par ferme laisse supposer que quelques paysans disposent en outre d'un cultivateur. La batteuse mécanique est totalement absente des groupes peu productifs (C et D), comme le sont d'ailleurs le râteau à cheval et la moissonneuse-faucheuse. Enfin, les groupes $\mathrm{C}$ et $\mathrm{D}$ possèdent deux fois moins de voitures légères que les autres groupes, détail révélateur lorsqu'on sait que le nombre de voitures a été introduit dans le recensement parce qu'on le considérait comme un excellent indicateur du niveau de richesse ${ }^{31}$.

Comme la valeur de l'équipement agricole ne figure que dans le recensement de 1861, nous avons calculé les moyennes de ces valeurs pour quelque 45 paysans qui occupaient à cette époque les lots à l'étude. Il appert que la valeur des instruments aratoires des Canadiens français ne dépasse pas 20 \$ par ferme alors que chez les Irlandais, les Écossais et les Anglais, elle atteint respectivement 50 \$, 39 \$ et 68 \$.

Il ressort donc que les deux groupes $(\mathrm{C}$ et $\mathrm{D})$, où la production est la plus faible et où les Canadiens français sont les plus nombreux, sont dotés de certains traits démographiques et économiques distinctifs et déterminants pour le choix de la stratégie agricole. Retenons, qu'en général ces paysans doivent nourrir un plus grand nombre de personnes à partir d'une terre plus réduite tout en bénéficiant d'une main-d'oeuvre et d'un outillage plus limités que les deux autres groupes (A et B). Il s'agit de familles plus jeunes qui affichent à tous les égards un niveau de richesse très inférieur à celui de leurs voisins des groupes $\mathrm{A}$ et $\mathrm{B}$. Nous avons en outre souligné que plusieurs de ces traits, notamment la jeunesse de la famille, la petitesse des terres et la pauvreté de l'outillage sont caractéristiques de l'ensemble des Canadiens français de notre échantillon. Voyons maintenant comment ces contraintes économiques et démographiques se conjuguent à celles du milieu physique pour orienter les stratégies agricoles.

\section{3 - Les stratégies sur sols argileux}

Les terres argileuses possèdent une bonne capacité d'échange cationique, c'est-à-dire que leur structure chimique permet de retenir

30 On rencontre plus de deux de ces instruments dans 22 pour cent seulement des fermes de l'échantillon et parmi les cinq agriculteurs qui en sont totalement dépourvus, figurent quatre Canadiens français.

31 Recensement du Canada, 1851-1852 (Imp. John Lovell, Québec, 1853), ix. 
quantité d'éléments nutritifs solubles, directement assimilables par la plante. Ces sols sont plus fertiles et plus propices à l'agriculture que les sols sableux. Toutefois, leur forte capacité de rétention d'eau et la compacité de leur structure présentent de graves inconvénients pour l'agriculteur. Elles sont lourdes et difficiles à travailler; les labours y sont ardus et doivent être effectués lorsque la terre a atteint un certain degré d'humidité: l'argile se transforme en bourbier lorsque trop humide et devient dure et impossible à retourner lorsque trop sèche ${ }^{32}$. Dans la région à l'étude, la plaine argileuse est périodiquement inondée par les eaux de la rivière Nation Sud, ce qui améliore la fertilité du sol grâce au dépôt de fines alluvions mais qui exacerbe les problèmes de drainage. Or, à l'époque, ces travaux représentaient de lourdes dépenses en termes de temps et d'argent ${ }^{33}$. En définitive, les argiles ont la capacité de soutenir une agriculture florissante à condition qu' on y investisse une quantité importante d'énergie sous forme de travail. L'intrant d'énergie constitue en quelque sorte le facteur limitatif de ce type de sol.

On l'a vu, sur le plan de la force de travail, les fermes canadiennesfrançaises du groupe $\mathrm{D}$ sont fort désavantagées par rapport à celles du groupe anglophone sur le même type de sol (A). Il s'agit, d'une part, de familles jeunes et nombreuses où peu d'enfants ont atteint l'âge de participer activement à l'exploitation familiale. Le paysan canadienfrançais et son épouse bénéficient en moyenne de l'aide supplémentaire de 0,6 personne tandis que le couple anglophone du groupe A peut compter sur celle de 2 individus en moyenne. D'autre part, il ne dispose que d'une machinerie réduite, ce qui accroît d'autant sa dépense énergétique. Son niveau de richesse manifestement moins élevé l'empêche très certainement d'embaucher une main-d'oeuvre d'appoint; or on connaît l'importance que peut avoir la disponibilité d'une force de travail accrue à certains moments stratégiques de la saison agricole. Dans de telles conditions, l'agriculteur canadien-français n'a d'autre choix que d'adopter une stratégie visant à minimiser l'intrant d'énergie. Cet objectif, imposé par sa condition familiale et matérielle, se reflète sur le type d'occupation du sol, le choix des cultures, les rendements et le cheptel.

Les Canadiens français du groupe D consacrent 35 pour cent de leur terre améliorée aux pâturages et aux prés de fauche contre 16 pour cent chez les anglophones du groupe A. Cette forme extensive d'oc-

32 E. A. Fitzpatrick, An Introduction to Soil Science (Edinburg, Oliver and Boyd, 1974).

33 R. E. Wicklund et N. R. Richards, Soil Survey of Russell and Prescott Counties (Guelph, Ontario Survey, report no. 33, 1962); E. Christie, A Centennial Story of Truth (Archives provinciales de l'Ontario, Mss. misc. collection 1860, ac 6000, 1962); K. Kelly, «The Artificial Drainage of Land in Nineteenth Century Southern-Ontario», Le géographe canadien, 19 (1975): 279298. 
cupation du sol ne permet évidemment pas une maximisation de la production; elle présente toutefois deux avantages majeurs: 1) elle est fort économique en termes d'énergie puisque les pâturages représentent la portion non ensemencée de l'espace amélioré ${ }^{34}$ et que les champs de foin n'exigent ni sarclage ni labour annuel. En ce sens, elle constitue donc un choix tout à fait adapté à la force de travail limitée de ce groupe. 2) Elle préserve la fertilité du sol grâce à un apport constant de matières organiques et assure ainsi l'équilibre du système écologique avec un minimum d'effort.

Il en découle qu'en moyenne, les Canadiens français du groupe $\mathrm{D}$ réservent moins d'espace aux plantes sarclées que les anglophones du groupe A, ce qui n'explique qu'en partie la faiblesse de leur production. En effet, ils parviennent à des rendements nettement inférieurs, du moins dans le cas de la pomme de terre, du foin et du blé (tableau 4). Puisqu'en outre ils doivent subvenir aux besoins d'une famille plus nombreuse, on peut s'attendre à ce que les surplus de production soient limités. Dans le cas du blé, par exemple, nous avons calculé que les surplus moyens des cultivateurs du groupe $\mathrm{D}$ équivalaient à un peu plus du tiers de celui des anglophones du groupe A qui consacrent pourtant en moyenne $3 / 4$ d'acre de moins à la culture de cette céréale ${ }^{35}$. Nous ignorons tout des méthodes de culture qu'utilisaient effectivement ces Canadiens français, mais tout porte à croire que l'outillage et la main-d'oeuvre limités imposaient le choix de méthodes économiques en termes de travail mais moins efficaces.

Lorsqu'on compare le nombre d'animaux domestiques dans les groupes $\mathrm{A}$ et $\mathrm{D}$, on se rend compte que les Canadiens français (D) possèdent moins de vaches laitières que les anglophones. En contrepartie ils élèvent plus de moutons et autant de cochons. A cette époque où l'industrie laitière commence à prendre le pas sur le commerce avec les chantiers, le nombre restreint de vaches du groupe $\mathrm{D}$ pourrait témoigner d'une certaine lenteur de la part des Canadiens français à s'adapter à la nouvelle conjoncture. Le niveau de richesse apparaît une fois de plus largement responsable de cet état de fait. En 1855, la valeur d'une vache laitière équivalait à celle de 10 moutons et de près de 4 cochons $^{36}$. Il est donc logique que les Canadiens français qui de toute évidence disposent de moyens financiers limités se consacrent plus volontiers à

34 Dans le recensement de 1871 , la surface en pâturages représente la différence arithmétique entre la surface améliorée et la surface cultivée. Canada, Recensement du Canada 18701871 (Ottawa, I. P. Taylor, 1873).

35 Nous avons utilisé les estimations fournies dans le recensement de 1851-52 où l'on évalue à 1,5 boisseau la quantité de blé nécessaire pour ensemencer 1 acre de terre et à 5 boisseaux la consommation annuelle par habitant, Canada, Recensement des Canadas pour 1851-1852, vol. 1, p. xxxi.

36 F. Lewis et M. McInnis, «Agricultural Output and Efficiency in Lower Canada, 1851», Research in Economic History, 9 (1984): 78. 
l'élevage du mouton et du cochon, animaux qui de surcroît n'exigent qu'un minimum d'entretien. Les vaches laitières imposent au contraire une quantité énorme de travail pour leur entretien, la traite et la transformation du lait en beurre ou en fromage. Puisque ces tâches incombaient surtout aux femmes, du moins jusqu'à ce que l'industrie laitière ne se commercialise ${ }^{37}$, on est en droit de se demander dans quelle mesure la faiblesse des effectifs féminins du groupe $\mathrm{D}^{38}$ a pu influencer la taille du cheptel laitier.

Quant aux chevaux, dont on connaît l'importance énergétique dans le système agricole de l'époque, ils sont en moyenne aussi nombreux chez les anglophones que chez les francophones. De plus, comme ces derniers labourent une proportion moindre de leurs terres, ils bénéficient en fait d'une force de travail animale supérieure (tableau 6). Il s'agit jusqu'à présent du seul avantage des Canadiens français sur le plan de la force de travail, à condition bien sûr que ces bêtes ne soient pas destinées à la vente ${ }^{39}$.

Moins limités par la quantité d'énergie disponible, les cultivateurs anglophones du groupe A ont, au contraire, opté pour une utilisation intensive du sol et une optimisation des rendements. La variété des récoltes et surtout la taille du cheptel laitier sont autant d'indices qui nous amènent à penser qu'il s'agit de personnes non seulement capables de percevoir l'ouverture de nouveaux marchés mais disposant des moyens nécessaires pour s'y engager. Ils continuent aussi de profiter des chantiers comme l'attestent les volumes des récoltes d'avoine, de foin et de pois. En outre, plusieurs d'entre eux produisent d'importantes quantités d'orge ${ }^{40}$ et à un plus faible degré de maïs ${ }^{41}$, deux cultures qui nécessitent un lourd investissement d'énergie. En définitive, ces cultivateurs retirent une plus grande quantité d'énergie du système sous

37 M. Cohen, «The Decline of Women in Canadian Dairying», Histoire sociale/Social History, 17,34 (1984): 307-335.

38 La main-d'oeuvre féminine chez les Canadiens français du groupe D équivaut aux deux tiers de celle des anglophones du groupe $\mathrm{A}$.

39 Il semble en effet qu'on ait élevé des chevaux pour le commerce avec les chantiers dans le comté de Prescott, R. L. Jones, History of Agriculture in Ontario, 119.

${ }_{40}$ Cette céréale était sans doute exportée dans les usines américaines de bière et de malt et constituait une bonne source de revenu. Le secrétaire du bureau d'agriculture affirme en effet que l'orge canadien, dont la qualité est fort appréciée des brasseurs américains, continuait d'être exportée malgré l'abrogation du traité de Réciprocité (Ontario Sessional Papers, IV, I, 19711972). Les argiles acides du comté de Prescott conviennent cependant fort mal à cette céréale exigeante et sa culture demandait un apprêt des terres coûteux en énergie (J. Dumanski et $\mathbf{J}$. Lendway-Zwickl, Climate and Soil Requirements for Economically Important Crop in Canada (Ottawa, Agriculture Canada, 1982), 36; B. E. Twamley, «A Living from the Land: a Brief History of Field Crops in Ontario», T. A. Growley, ed., First annual agricultural history of Ontario seminar proceedings (Guelph, 1976), 81).

41 Le maïs était produit avec parcimonie à cause de la quantité de travail énorme qu'encourait sa culture; c'est du moins ce que laisse entendre R. Russell, North America, its Agriculture and Climate (Edinburgh, 1857), 240. 
forme de produits végétaux (récoltes) ou animaux (viande, lait, beurre etc), mais il ne faut pas oublier qu'ils y ont également investi une plus grande quantité d'énergie sous forme de travail et d'engrais.

Le calcul de l'efficacité écologique de ces deux groupes, c'est-àdire le rapport entre l'intrant ${ }^{42}$ et l'extrant d'énergie, aurait permis d'évaluer dans quelle mesure la moindre production des Canadiens français est proportionnelle à la moindre quantité d'énergie investie. Malgré cette limite inhérente aux sources disponibles, nos résultats montrent clairement que sur les sols argileux, le groupe de fermes le moins productif correspond à celui où l'intrant d'énergie est le plus faible.

\section{4 - Les stratégies sur sols sableux}

Au contraire des argiles dont la structure en feuillets permet d'emmagasiner l'eau et de fixer les éléments nutritifs, les sables sont principalement formés de grains de quartz dont la structure chimique compacte interdit la fixation de cations et la rétention d'eau. La fertilité et la capacité de rétention d'eau des sols sableux provient donc essentiellement de la matière organique qu'ils contiennent. En revanche, il s'agit de sols légers, faciles à travailler. Le facteur limitatif n'est donc plus l'intrant d'énergie mais bien la quantité de nutriments et d'eau disponible dans le sol. Dans ce type d'environnement la stratégie agricole la plus appropriée est de pratiquer une agriculture extensive où une forte proportion de l'espace est annuellement réservée à la production de fourrage (pâturage et foin). A condition de ne pas être totalement exportés à l'extérieur du système, ces herbages fournissent au sol une quantité appréciable de matière organique sous forme de fibres végétales ou de fumiers.

L'utilisation extensive des sols pratiquée par les cultivateurs du groupe B s'avère donc bien adaptée aux conditions du milieu. Avec une surface améliorée de 58 acres, ces derniers peuvent facilement se permettre d'allouer la moitié de leurs terres aux herbages et de préserver ainsi la teneur du sol en nutriments. Il leur reste encore une trentaine d'acres pour produire les céréales et les racines nécessaires à leur alimentation et à celle du bétail. Mais comment, sur une terre de 36 acres, les cultivateurs canadiens-français et écossais du groupe $C$ peuvent-ils concilier les exigences du milieu avec celles plus pressantes de leur propre subsistance? Ils doivent forcément ensemencer un minimum de terre pour subvenir à leurs besoins de sorte que l'espace attribué aux herbages s'en trouve considérablement restreint; il équivaut en fait au cinquième de la surface améliorée.

42 L'intrant d'énergie est représenté par la force de travail humaine, la machinerie, les fumures et autres détritus organiques. 
En effet, on remarque que les paysans du groupe $\mathrm{C}$ affectent en moyenne 29 acres aux cultures sarclées, soit à peine un acre de moins que le groupe $\mathrm{B}$. Pourtant les récoltes sont considérablement inférieures pour toutes les cultures sans exception (tableau 5). Le tableau 4 montre que les soles de blé et de pommes de terre du groupe $\mathrm{C}$ ont un rendement moyen équivalant à près de la moitié de celui du groupe B. Seuls les champs de foin se montrent aussi généreux. La pauvreté du groupe $\mathrm{C}$ se manifeste également par la taille du troupeau. La taille réduite des troupeaux bovin et ovin, en particulier, incite à penser qu'il leur est impossible d'enrichir le sol avec une quantité adéquate de fumures.

Prenons l'exemple de John Shaw qui possède une terre de 100 acres sur la 9e concession du canton de Plantagenet et de son voisin Antoine Rochon dont la terre de 50 acres est située sur le même type de sol sableux. La famille Shaw compte, en 1871, 6 garçons et 3 filles de 14 ans et plus. Natif d'Irlande, John Shaw a défriché 60 acres dont 15 sont laissés en pâture et 15 autres en foin. Sur les 30 acres restants, il produit 60 boisseaux de blé, 600 d'avoine, 40 de pois et 300 de pommes de terre. Grâce à une utilisation extensive du sol et à un fumage des terres que l'on suppose abondant vu la taille du troupeau ovin et bovin ( 6 vaches laitières, 3 boeufs, 17 moutons), il obtient des rendements remarquables de blé ( 20 boisseaux à l'acre) et de pommes de terre (100 boisseaux à l'acre). De plus, 80 livres de laine et 400 livres de beurre ont été fabriquées à la ferme cette année-là. Les Rochon ont également une famille nombreuse, 7 enfants, mais tous au-dessous de 14 ans. Des 30 acres améliorés, 5 seulement servent au pâturage et une seule est réservée au foin. A l'instar de Shaw, Antoine Rochon cultive blé, avoine, pois et pommes de terre; il ajoute cependant la culture du seigle et du sarrasin, deux espèces adaptées aux sols ingrats ainsi que celle du tabac qui, dans la région à l'étude, est l'apanage des Canadiens français ${ }^{43}$; la production est ici plus variée mais considérablement moins abondante que chez les Shaw. A titre de comparaison, la famille Rochon a récolté 6 fois moins d'avoine, 7 fois moins de pois et 2 fois moins de pommes de terre. Le cheptel est également bien pauvre: 2 vaches, 1 boeuf, 2 moutons et 3 cochons. Rien d'étonnant donc à ce que cette petite ferme dont les $4 / 5$ de l'espace sont constamment cultivés et dont le sol est manifestement mal engraissé, obtienne de piètres rendements.

Bien que les effets d'un tel mode de culture se fasse sentir plus vite sur les sols sableux où la fertilité du sol constitue le principal facteur limitatif, elle ne manque pas de se manifester à plus ou moins brève échéance sur les argiles aussi. Nous trouvons d'ailleurs dans ce groupe (C) quelques petites fermes sur argile (5 dont 4 appartiennent à des Canadiens français) qui affichent les mêmes caractéristiques.

43 Dans l'échantillon à l'étude, le tabac est surtout cultivé par les Canadiens français, qui en ont produit en moyenne de 10 à 15 livres par ferme. 
Bref, le type d'agriculture pratiqué par les Canadiens français et les Écossais du groupe $\mathrm{C}$ se montre peu adapté aux conditions du milieu. Il semble toutefois que la petitesse des terres, la faible taille du troupeau ainsi que la pauvreté de l'outillage et de la main-d'oeuvre n'offrent d'autre choix. Il s'agit d'une stratégie vouée à la faillite puisqu'elle consiste à extraire le maximum d'un milieu écologiquement fragile tout en y investissant peu d'énergie. Cette stratégie rallie en somme les fermiers les plus démunis, propriétaires de terres exiguës. Les Canadiens français y sont nombreux parce qu'ils se particularisent généralement par un niveau de richesse peu élevé et par des terres de faible dimension ${ }^{44}$.

\section{CONCLUSION}

Les cultivateurs canadiens-français du comté de Prescott sont, nous l'avons vu, confrontés à des contraintes sociales bien spécifiques. Conjuguées à certaines caractéristiques du milieu physique, ces contraintes n'autorisent pas la mise en oeuvre d'une stratégie visant à une optimisation de la production. Sur les argiles, ces paysans ont développé un système de culture qui leur est propre et qui, tout traditionnel qu'il ait pu paraître à Jones, s'avère bien adapté aux exigences de l'environnement physique et social: par une occupation extensive du sol, ils réussissent d'une part à réduire l'intrant d'énergie qui constitue leur principal facteur limitatif et d'autre part à se garantir, à peu de frais, contre la déplétion rapide du sol. Sur les sables, leur comportement est très peu différent de celui des anglophones. Sur une terre suffisamment vaste, ils peuvent se permettre une utilisation très extensive de l'espace qui convient bien à ces sols pauvres. On les retrouve cependant en plus grand nombre sur des terres exiguës où ils se voient alors forcés d'adopter, à l'instar de leurs confrères écossais, une stratégie de survivance et de compromettre ainsi la pérennité du système.

Il a été impossible de calculer les flux énergétiques et l'efficacité écologique de ces agro-écosystèmes, faute de données suffisantes. Toutefois, les données relatives à la population et au niveau de richesse ont fourni de solides raisons de croire à un écart minime entre l'efficacité écologique des quatre groupes étudiés. En effet, les deux groupes (C et D) où les Canadiens français sont nombreux produisent moins d'énergie

\footnotetext{
44 Cette dernière particularité a également été observée par Little chez les francophones des Cantons de l'Est. «The French Canadians within the two townships concentrated upon the same major products as the anglophones, but their crop yields usually were not as high. Weither or not this was caused by a more careless agricultural practices or simply by inferior land, the primary handicap of the French Canadians was the smallness of their farms». J. J. Little, «The Social and Economic Development of Settlers in two Quebec Townships, 1851-1870», D. H. Akenson, ed., Canadian Papers in Rural History (Gananoque (Ont.), Langdale Press, 1982), 3: 98.
} 
(récoltes, produits d'origine animale) que les groupes A et B mais en contrepartie, ils investissent moins de travail et d'engrais. Notre analyse a, en outre, clairement montré que la maximisation de la production ne constitue pas dans tous les cas une stratégie possible ni même souhaitable. En définitive, ces paysans canadiens-français semblent tirer le meilleur parti possible du sol et des ressources parfois très modestes dont ils disposent ${ }^{45}$.

45 En cela, le paysan canadien-français du comté de Prescott se rapproche du modèle de l'habitant développé par Wallot et Paquet: «... un être rationnel, armé comme tous les autres agents économiques, d'une rationalité limitée... et soumis, dans son processus de prise de décision, aux contraintes normales imposées par les coûts de décision et de transaction ainsi que par des circonstances du milieu ambiant. Comme les contraintes auxquelles l'habitant est confronté peuvent être différentes de celles auxquelles font face ses compatriotes anglophones, ses comportements pourront s'avérer autres: non pas par conservatisme inné, mais parce que le contexte différent commande une stratégie différenciée.» G. Paquet et J.-P. Wallot, «Stratégie foncière de l'habitant: Québec (1790-1835)», RHAF, 39,4 (printemps 1986): 555. 